Publisher: Taylor \& Francis

Journal: Ergonomics

DOI: http://dx.doi.org/10.1080/00140139.2016.1158323

\title{
The impact of false warnings on partial and full lane departure warnings effectiveness and acceptance in car driving
}

Running head: Imperfect lane departure warnings

Jordan Navarro ${ }^{a}$, Elsa Yousfi ${ }^{\mathrm{a}}$, Jonathan Deniel ${ }^{\mathrm{a}}$, Christophe Jallais ${ }^{\mathrm{b}}$, Mercedes Bueno ${ }^{\mathrm{b}}$, and Alexandra Fort ${ }^{\mathrm{b}}$

${ }^{a}$ Laboratoire d'Etude des Mécanismes Cognitifs (EA 3082), University Lyon 2, Bron, France

${ }^{b}$ LESCOT-TS2-IFSTTAR (French Institute of Science and Technology for Transport, Development and Networks), Bron, France

Address correspondence to Jordan Navarro, Institut de Psychologie, 5 avenue Pierre Mendès-France, 69676 Bron Cedex, France, jordan.navarro@univ-lyon2.fr 


\section{The impact of false warnings on partial and full lane departure warnings effectiveness and acceptance in car driving}

In the past, lane departure warnings were demonstrated to improve driving behaviours during lane departures but little is known about the effects of unreliable warnings. This experiment focused on the influence of false warnings alone or in combination with missed warnings and warning onset on assistance effectiveness and acceptance. Two assistance unreliability levels (33\% and 17\%) and two warning onsets (partial and full lane departure) were manipulated in order to investigate interaction. Results showed that assistance, regardless unreliability levels and warning onsets, improved driving behaviours during lane departure episodes and outside of these episodes by favouring better lane keeping performances. Full lane departure and highly unreliable warnings, however, reduced assistance efficiency. Drivers' assistance acceptance was better for the most reliable warnings and for the subsequent warnings. The data indicate that imperfect lane departure warnings (false warnings or false and missed warnings) further improve driving behaviours compared to no assistance.

Practitioner summary: This paper revealed that imperfect lane departure warnings are able to significantly improve driving performances and that warning onset is a key element for assistance effectiveness and acceptance. The conclusion may be of particular interest for lane departure warning designers.

Keywords: lane departure warning; warning onset; false alert; alert miss; steering behaviour 


\section{Introduction}

Road transport-related accidents continue to be one of the leading causes of disabilities and deaths globally. The last World Health Organization report on that matter indicated that the total number of road traffic deaths was at 1.24 million per year through the world (World Health Organization, 2013). Several approaches have been investigated and combined to tackle this major issue, including speed limits reduction, increase in seatbelt use and reduction of drunk driving (World Health Organization, 2013). Another approach consisted of assisting drivers while driving so as to help them attain higher performances and reduce the number of road accidents. A first attempt to do so consisted in the use of warning signs (Dewar, 1993; Lehto, 2000) with mixed results (Carson \& Mannering, 2001; Charlton, 2006, 2007), calling in question the effectiveness of road hazard warning signs (Dewar, 1988; Fisher, 1992; Johansson \& Backlund, 1970; Macdonald \& Hoffmann, 1991). The introduction of in-vehicle assistance devices offers much more perspectives (Dewar, 1993) because those devices take into account the dynamic driving environment and drivers' actions.

Navigation, hazard identification and vehicle control are the three main sub-tasks drivers are performing sequentially or in parallel while driving (Stanton, Young, Walker, Turner, \& Randle, 2001). Navigation refers to drivers' ability to move from one point to another within the road network. Wellknown assistances have been imagined to help drivers navigating in particular using global positioning systems to locate the vehicle position on an electronic map. Hazard identification can also be assisted for instance with parking aids emitting increasingly frequent beeps when approaching an obstacle. Finally and directly linked with the reported work, both longitudinal and lateral dimensions of vehicle control may be assisted.

Longitudinal control assistances such as cruise control and forward collision warning systems are the oldest and most common vehicle control assistances currently available on cars (Shladover, 1995; Vahidi \& Eskandarian, 2003). The number of assistances aimed to help drivers in their lateral control performance has also constantly grown in the last few decades (Tango \& Montanari, 2006). Although questioned recently for collision avoidance assistance, the human-centred automation principle usually requires the driver to have the final authority over the automation (Itoh \& Inagaki, 2014). A comprehensive review of lateral control assistance devices from a human centred perspective (Navarro, Mars, \& Young, 2011) based on a classification from a mixture of two theoretical frameworks (Hoc, Young, \& Blosseville, 2009; Young, Stanton, \& Harris, 2007) is available. Among the several human- 
machine cooperation modes reported, the "warning mode" is the most frequently used as far as the assistance device sought interacts with the driver. Often referred to as Lane Departure Warning Systems (LDWS), those devices warn drivers when the vehicle position on its lane is judged as hazardous. LDWS have been revealed to be effective in several studies by improving the steering wheel response and reducing the time needed to recover a safe position on the driving lane (Hoc et al., 2006; Navarro, Mars, Forzy, El-Jaafari, \& Hoc, 2010; Navarro, Mars, \& Hoc, 2007; Suzuki \& Jansson, 2003). Among the different sensorial modalities used to convey the warning, auditory warning similar to rumble strip noise is preferred by drivers (Kozak et al., 2006; Maltz \& Shinar, 2004; Navarro et al., 2010; Tijerina, Jackson, Pomerleau, Romano, \& Petersen, 1996; Ziegler, Franke, Renner, \& Kühnle, 1995). This may be accounted for by drivers' familiarity with rumble strips available for years on a number of roads (Navarro et al., 2010). In addition the immediacy of auditory warnings may also favour drivers' preference (Edworthy, Stanton, \& Hellier, 1995). It may be observed that while LDWS were always reported as being effective, drivers did not appear very enthusiastic about the use of LDWS. Drivers were seen as lukewarm about the LDWS concept after their subjective evaluation (Tijerina et al., 1996).

Like any assistance device a perfect level of reliability cannot be guaranteed with LDWS. If LDWS are expected to deliver Correct Warnings (CW), those devices are not perfectly reliable and might also deliver Incorrect Warnings (IW) in some cases. Unreliability is defined here as the ratio between IW and CW expressed as a percentage. This imperfect reliability is related to the extreme variety of situations that might be found while driving such as poor weather conditions, road surface alteration, unexpected objects or presence of liquid on the road surface, missing or damaged lane marking, or assistance captor malfunction. Two types of errors might be observed: (1) False Warnings (FW) when the assistance device triggers a warning when the situation did not require this and (2) Missed Warnings (MW) when the assistance device should have triggered a warning but failed to do so. If the effectiveness and acceptance of LDWS are relatively well documented in the literature, surprisingly very little is known about the influence of LDWS errors on drivers' behaviours. This experiment focuses on False Warnings alone or in combination with Missed Warnings.

To the best of our knowledge only one simulator and one test-track study was performed to evaluate reliable versus unreliable auditory LDW impact on driving behaviours (Rudin-Brown \& Noy, 2002). For the unreliable condition the LDW provided both FW (every third warnings) and MW (every seven minutes in a thirty minute driving scenario) while the LDW was perfectly reliable in the reliable 
condition. Lane keeping performances were improved regardless of whether the assistance device was reliable or not as drivers approached the lane-edge zones less often with an LDW than without. Drivers' trust in the device grew after exposure to the assistance and this increase was greater for the perfectly reliable compared to the unreliable assistance. Those data are in line with the similar improvement of time headway (i.e. increase) recorded with both reliable (100\%) and unreliable $(95 \%, 80 \%, 60 \%$ reliable) in-vehicle collision avoidance warning systems devoted to assist longitudinal control of the vehicle (Benyaacov, Maltz, \& Shinar, 2002; Maltz \& Shinar, 2004, 2007). Nevertheless, lane departure episodes were not analysed in the Rudin-Brown \& Noy experiments, leaving the question of LDW errors effects on subsequent lane departure without response.

If the current study aimed to gain a better understanding of how assistance errors (FW or FW and MW) may impact following lane departures, data collected from in-vehicle collision avoidance warning systems provide useful insights. Some experimental results are in line with common sense indicating that unreliable assistance are not as effective as reliable assistance in particular with false warnings (Bliss \& Acton, 2003; Enriquez \& MacLean, 2004; Maltz \& Shinar, 2004). For in-vehicle collision avoidance warning systems with both false and missed warnings, it was reported that only false warnings significantly modified drivers' behaviours by triggering inappropriate speed reduction (Maltz \& Shinar, 2004). Another unreliable collision warning system, that committed both false and missed warnings, led to longer reaction times for a lead car braking compared to a perfectly reliable assistance (Abe, Itoh, \& Tanaka, 2002). Longer reaction times were observed for improperly warned events and for the following events properly warned (i.e. lead vehicle brake events). This indicates that unreliable assistance was not only negative for the situations improperly warned but was also contaminating subsequent events.

Interestingly not all experimental results revealed-the best driving performance with a perfectly reliable assistance. Several authors reported some drivers might have been complacent and relied too much on a perfectly reliable assistance device. This may result in longer reaction times, degradation of the braking response and even more crashes (Abe et al., 2002; Bliss \& Acton, 2003; Maltz \& Shinar, 2007). Finally, other studies indicated no significant effect of unreliable assistance (false and missed warnings) at various reliability levels compared to reliable assistance when looking at true warnings (Ben-yaacov et al., 2002; Bueno, Fabrigoule, Deleurence, Ndiaye, \& Fort, 2012; Maltz \& Shinar, 2004). Reliability of longitudinal control assistances on driving performances are not perfectly clear and are 
probably context dependent (Lees \& Lee, 2007). However, it appears that false warnings are able to modify driving behaviours and might also be judged as less acceptable because they intrude more often on driver activity. In that regard, false warnings were found to diminish trust in and compliance with collision warning systems (Lees \& Lee, 2007).

In practice the effects of assistance reliability cannot be considered independently from warning onset. The earlier the warning the more frequent the risk of false warning and the later the warning the more frequent the risk of missed warning. Indeed LDWS delivers warnings when a given position on the driving lane is reached. The earlier the warning the most central the position of the vehicle is on its lane. In practice and from drivers' perspective, with a too early warning onset, some warnings would be delivered even when drivers judge their position in the lane as non-hazardous, resulting in an increase of false warnings. At the reverse and still from driver perspective, if the warning is delivered too late, drivers might initiate a correction of the vehicle trajectory and receive the warning after that initiation or even no warning when the correction is very efficient, resulting in an increase of missed warnings. If warning onset is suspected to be a key component of both effectiveness and acceptance of LDWS (Navarro et al., 2011; Werneke, Kleen, \& Vollrath, 2013) only a single experiment directly manipulated the lane departure warning onset so as to assess its effects on drivers' performance (Tijerina et al., 1996). It came as little surprise that early warnings gave rise to fewer lane departures but more warnings and lower acceptance than late warnings. Early warnings provide drivers with sufficient time to adjust their driving behaviour so that unsafe last moment reactions can be avoided (Werneke et al., 2013). However, as said before, the earlier the warning the more likely it is that it will be considered a false warning (McGehee, Brown, Lee, \& Wilson, 2002; Parasuraman, Hancock, \& Olofinboba, 1997; Shinar, 1978). Those warning signals might then be judged as annoying and useless, leading drivers to ignore them (Abe \& Richardson, 2004, 2006).

Early and late rear-end collision warnings were shown to reduce the number of collisions for distracted and non-distracted drivers (Lee, McGehee, Brown, \& Reyes, 2002). Distracted drivers responded more quickly with the assistance and early warnings reduced the number of collisions by more than $80 \%$ while late warnings reduced collisions by $50 \%$. Several studies dealing with forward collision warning systems indicated that early warnings were more effective and trusted than late warnings especially for emergency braking (Abe \& Richardson, 2004, 2005, 2006). In short, it seems that warnings should be delivered as early as possible to improve performances and trust but without increasing the 
objective and/or subjective false warning rate. An interaction between warning onset and assistance reliability (false warnings in particular) can be expected. In line with previous results, it can be hypothesized that early onset combined with false warnings will lead to poorly efficient and acceptable assistance. The adjunction of missed warnings to false warnings should lead to a more important decrease in driver subjective reliability assessment that may have an impact on driving behaviour. A warning may be triggered more or less early but the notion of early or late is relative to a reference (e.g. lane marking). To describe as accurately as possible the warning onsets used in this experiment, the terms "Partial lane departure" and "Full lane departure" were preferred to the early and late warning terminology.

Assistance was expected to improve driving performances during lane departure episodes compared to unassisted driving. Partial lane departures were expected to elicit better recovery manoeuvres during lane departure episodes than Full lane departures. But the main objective of this experiment was to assess the impact of false warnings alone or mixed with missed warnings on subsequent lane departure episodes in terms of assistance effectiveness and acceptance. It was hypothesized that the less reliable the assistance is the less effective and acceptable it would be. The warning onset (Partial or Full lane departure) was expected to interact with lane departure warning reliability. Partial lane departure warnings associated with false warnings should be judged as poorly acceptable and, therefore, lead to the worst driving performances. Another objective was to evaluate how assistance reliability and onset influence driying behaviour outside of lane departure episodes.

\section{Method}

\subsection{Participants}

Forty-eight participants ( 24 females) divided into four groups of twelve participants, aged from 24 to 45 years $($ mean age $=31$ years \pm 5 ), with driving experience ranging from 4 to 24 years $($ mean $=11.2$ years \pm 5.5 ) and driving from 500 to 50,000 kilometres per year (mean $=12,400$ kilometres \pm 11000 ) took part in the experiment. None experienced simulator sickness and each received 50 euros for their participation.

\subsection{Driving simulator}

A fixed-base simulator built on a Peugeot 308 cabin equipped with a manual gearbox, a force feedback steering wheel and brake, accelerator and clutch pedals developed by IFSTTAR-COSYS-LEPSIS was used for the experiment. The visual environment, consisting of a model of a straight three-lane road 
developed for the purpose of the experiment, was projected onto seven screens providing a horizontal angle of about $300^{\circ}$ around the driver.

\subsection{Lane Departure Warning (LDW)}

The Lane Departure Warning (LDW) emitted a rumble strip sound played from the loudspeakers of the simulator cabin when the vehicle was leaving its lane. The auditory warning was audible as long as the car was not driven back into its lane.

Two warning onsets (Partial Lane Departure onset -Partial LD- and Full Lane Departure onset -Full LD-) were used. With the Partial LD onset the lane departure warning was triggered when a front wheel of the vehicle entered into contact with a lane marking whereas with the Full LD onset the front wheel had to cross the lane marking completely.

Two groups of participants were associated with an unreliability level of $17 \%$ and the two other groups with an unreliability level of 33\%. For each unreliability level, a group only faced False Warnings and the other group faced both False and Missed Warnings. Unreliability levels were selected based on previous experiments since reliability levels under 60\% were showed to be ineffective (Dingus et al., 1997). And studies on FCWS reliability effects used reliability levels of between 60 and 95\% (Maltz \& Shinar, 2004, 2007).

In order to provide control conditions, driving scenarios without lane departure warning were included for each participant. Hereafter drives with the LDW will be refereed as Assistance condition (A) and drives without LDW as No Assistance condition (NA). The comparison between A and NA conditions was set to contrast driving behaviours with LDW versus without LDW.

\subsection{Distraction task}

Lane departures were brought about by means of a reading task of words displayed on a 7-inch screen placed on the dashboard $\left(25^{\circ}\right.$ down and $35^{\circ}$ right from the driver gaze straight ahead). The screen displayed four words refreshed every second among the 3097 selected from the MANULEX database (Lété, Sprenger-Charolles, \& Colé, 2004) based on their length (between 6 and 9 letters) and frequency of use (50\% of the words the closer to the frequency average). Between 1 and 4 infinitive verbs were presented every eight words displayed. The distraction task was run while driving and participants were instructed to read aloud as many verbs as possible. Participants were also asked not to look at the road 
and not to turn the steering wheel during distraction tasks. Experimenters ensured that participants did so using cameras located in the driving simulator.

For all driving scenarios except training, distraction tasks were separated from each other by a randomised time in the range of 20 to 40s. Half the distraction tasks did not lead to lane departure and half did (equally distributed between left and right lane departures). To ensure that the distraction tasks led to a left or a right departure or to no lane departure, vehicle dynamics were virtually manipulated during the reading task. To do this the simulated vehicle heading was manipulated virtually; those manipulations had no effects on the steering wheel position. It was not possible for participants to notice those manipulations and none did. For those distraction tasks that should not lead to lane departure, vehicle trajectory was maintained into the driving lane and the distraction task was programmed to last from 4 to 8 s. For those distraction tasks that should lead to lane departure, vehicle trajectory was slightly modified to the right or left to ensure a right or left lane departure, respectively.

When the distraction task stopped, the 7-inch screen used to display the words remained black. In the case of a driving scenario with driving assistance (A), the sound of the lane departure warning was played at the same time. In the case of a driving scenario without assistance (NA), the distraction task stopped when the vehicle reached the same Partial or Full lane departure positions used with the LDW.

Traffic in the two other driving lanes and oncoming traffic were present at a rate of approximately six vehicles per kilometre. However, the traffic was arranged in such a way that drivers never had to take into account a potential risk of collision nor change lane.

\subsection{Procedure}

Drivers were instructed to drive on the middle lane of the three-lane road, to keep both hands on the steering wheel and to reach the speed of $90 \mathrm{KPH}$. When the speed of $90 \mathrm{KPH}$ was reached, cruise control was automatically engaged and the same speed kept for the rest of the driving scenario.

After a training scenario aimed to ensure a participants' familiarisation with the instructions, the driving simulator and the distraction task, participants took part to four different driving scenarios. These driving scenarios were followed by questionnaires: (a) the NASA-TLX in order to assess mental workload (Hart \& Staveland, 1988) and (b) a questionnaire of assistance device acceptance (Reagan \& Bliss, 2013).

In practice each participant took part in four successive driving scenarios balanced in each group of participants using a Latin square design (1) No Assistance with partial lane departure, (2) No 
Assistance with full lane departure, (3) Assistance with partial lane departure, and (4) Assistance with full lane departure. A given participant kept the same unreliability level (17\% or 33\%) in the presence of the LDW and faced the two onsets (Partial LD and Full LD).

Therefore the driving scenarios 1 and 2 were common to all participants but scenarios 3 and 4 differed from one group of participants to the other. LDW errors according to the group of participants were as follows:

- The first group associated with a LDW unreliability of 2/6 (33\%) faced two False Warnings and four Correct Warnings.

- The second group associated with a LDW unreliability of 2/6 (33\%) faced one False Warning, one Missed Warning and four Correct Warnings.

- The third group associated with a LDW unreliability of $1 / 6(17 \%)$ faced one False Warnings and five Correct Warnings.

- The fourth group associated with a LDW unreliability of 2/12 (17\%) faced one False Warning, one Missed Warning and ten Correct Warnings.

In the two unassisted driving scenarios all participants faced twelve lane departure events, six in the no assistance Partial LD drive and six in the no assistance Full LD drive. In the two assisted driving scenarios (assistance with Partial LD and assistance with Full LD), each participant of group 1 and 2 faced twelve lane departure events with eight Correct Warnings and four Incorrect Warnings. Each participant of group 3 faced twelve lane departure events with ten Correct Warnings and two Incorrect Warnings. Each participant of group 4 faced twenty-four lane departure events with twenty Correct Warnings and four Incorrect Warnings.

So as to keep the duration of the experiment acceptable only the last group 4 had longer (16 minutes) driving scenarios with assistance compared to other driving scenarios (8 minutes). The LDW errors occurred at the second or third distraction task (and first lane departure episode) and at the fifth or sixth distraction task (and third lane departure episode) when two LDW errors were planned. A given participant kept the same unreliability level (17\% or 33\%) in the presence of the LDW and faced the two onsets (Partial LD and Full LD).

If participants were presented with the sound of the assistance device and the way it works prior to the experiment, they did not experience the LDW device before the experiment. Moreover, they were 
not informed about the assistance device reliability or onset manipulations. The approximate duration of the experiment was 75 minutes.

\subsection{Data analysis}

\subsubsection{Objective assessment}

In order to assess driving behaviours during lane departure episodes objectively, the Duration of Lateral Excursion (DLE) and Steering Reaction Time (SRT) were computed. DLE was the time spent by the most outward portion of the vehicle outside of the driving lane during a provoked lane departure. SRT was the time elapsed between the end of the distraction task (and warning trigger if a LDWS is present) and the moment when drivers began to turn the steering wheel. In order to evaluate lateral control outside lane departure episodes, the surface between the lane centre and the centre of the car was computed for all road sections with no distraction tasks and associated lane departures.

Newman-Keuls tests were used for post-hoc comparisons. The level of statistical significance of $P<0.05$ was used in all tests.

2.6.1.1 Impact of False Warnings and False Warnings plus Missed Warnings on subsequent lane departures

A $2 \times 2 \times 2 \times 2$ mixed design ANOVA with two between-participants factors (unreliability level: $17 \%, 33 \%$ and LDW error type: false warning, false and missed warnings) and two within-participants factors (driving assistance: no assistance, assistance and lane departure onset: Partial LD, Full LD) was used to assess the impact of LDW errors on subsequent lane departures.

\subsubsection{Lane departure following a False or a Missed Warning}

A $8 \times 2$ mixed design ANOVA with one between-participants factor (lane departure onset Partial LD, Full LD) and one within-participants factor (lane departure occurrence: after the FW of group 17\%, after the two FWs of group 33\%, after the FW and the MW of group 17\%, after the FW and the MW of group $33 \%$ and in the no assistance condition) was used to assess behaviours for the lane departure immediately following a LDW error as compared to no assistance condition.

\subsubsection{Lateral position on the lane outside lane departure episodes}


A $2 \times 2 \times 2 \times 2$ mixed design ANOVA with two between-participants factors (unreliability level: $17 \%, 33 \%$ and LDW error type: false warning, false and missed warnings) and two within-participants factors (driving assistance: no assistance, assistance and lane departure onset Partial LD, Full LD) was used to assess driving behaviours outside of the distraction tasks and lane departures episodes.

\subsubsection{Subjective assessment}

\subsubsection{Mental workload}

The same $2 \times 2 \times 2 \times 2$ mixed design ANOVA with two between-participants factors (unreliability level: 17\%, 33\% and LDW error type: false warning, false and missed warnings) and two within-participants factors (driving assistance: no assistance, assistance and lane departure onset Partial LD, Full LD) was used to evaluate subjective mental workload across conditions based on the six dimensions of the NASA-TLX questionnaires.

\subsubsection{Assistance acceptance}

Finally, a 2 × 2 × 2 mixed design ANOVA with one between-participants factor (lane departure onset Partial LD, Full LD) and two within-participants factors (unreliability level: 17\%, 33\% and LDW error type: FW, FW and MW) was computed for each of the eight dimensions of the acceptance questionnaire (reliable, predictable, trustworthy, acceptable, pleasing, annoying, accurate, agreeable). Each of those dimensions was evaluated on a scale from 0 to 10 (Reagan \& Bliss, 2013).

\section{Results}

\subsection{Objective assessment}

3.1.1 Impact of False Warnings and False Warnings plus Missed Warnings on subsequent lane departures

Duration of lateral excursion (DLE). Mean DLE decreased significantly with the LDW assistance compared to the no assistance condition (assistance: $4.7 \mathrm{~s}$, no assistance: $5.75 \mathrm{~s}, F(1,44)=102, p<.001, \eta^{2}$ $=0.70$, see Fig. 1A). The onset of the LDW also impacted significantly on DLEs (Partial LD: 4.93s, Full LD: $\left.5.52 \mathrm{~s}, F(1,44)=48.16, p<.001, \eta^{2}=0.52\right)$. If LDW unreliability failed to attain the statistical significance level $\left(33 \%: 5.5 \mathrm{~s}, 17 \%: 4.95 \mathrm{~s}, F(1,44)=3.89, p=.05, \eta^{2}=0.08\right)$, a statistically significant interaction was found between LDW unreliability and the lane departure onset $\left(F(1,44)=4.87, p<.04, \eta^{2}\right.$ 
$=0.1$, see Fig. 1B). Post-hoc analyses revealed that Full LD onset with the assistance unreliable at $33 \%$ resulted in significantly longer DLEs (5.34s) than Partial LD onset at the same unreliability level and the $17 \%$ unreliable assistance regardless of onset (mean $4.49 \mathrm{~s}$ ). In short, DLEs tend to be longer at a $33 \%$ assistance unreliability level than at a $17 \%$ unreliability level, especially for Full LD onsets.

$<$ Insert Figure 1 about here >>

Steering reaction time (SRT). The LDW device unreliability and the type of error did not significantly affect mean SRTs $F(1,44)=0.82, p=.37$ and $F(1,44)=0.15, p=.7$, respectively). However, mean SRTs were significantly reduced in the presence of the assistance device compared to the no assistance condition (assistance: $0.93 \mathrm{~s}$, no assistance: $1.26 \mathrm{~s}, F(1,44)=61.99, p<.001, \eta^{2}=0.58$ ). In addition, the onset also impacted significantly on SRTs (Partial LD: 0.97s, Full LD: $1.22 \mathrm{~s}, F(1,44)=34.98, p<.001, \eta^{2}$ $=0.44)$. Finally, a statistically significant interaction between whether or not assistance was present and onset was found $F(1,44)=4.55, p<.04, \eta^{2}=0.09$, see Fig. 2). If SRTs were shorter in the presence of the assistance this reduction was greater with Partial LD onsets (no assistance - assistance difference: $0.4 \mathrm{~s}$ ) than with Full LD onsets (no assistance - assistance difference: 0.26s).

$<$ Insert Figure 2 about here >>

An overview of those results has been summarized in Table 1 by crossing the four driving scenarios and the four groups of participants (see procedure section).

<<Insert Table 1 about here >

\subsubsection{Lane departure following a False or a Missed Warning}

Duration of lateral excursion (DLE). A significant main effect of an error from the assistance (FW or MW) on the following lane departure and compared to no assistance DLE was revealed by the ANOVA $\left(F(7,88)=3.08, p<.01, \eta^{2}=0.2\right.$, see Fig. 3). Post-hoc analyses indicated that only the lane departure episode immediately following the $\mathrm{FW}$ in the $17 \%$ group and the $\mathrm{MW}$ in the $17 \%$ group $\mathrm{FW}$ and $\mathrm{MW}$ differed significantly from the no assistance condition. For those two lane departures DLE was reduced compared to no assistance even just after one error from the assistance. All other lane departures immediately following an assistance error gave rise to insignificantly shorter DLE than those observed in the no assistance condition. The lane departure onset did not significantly change DLE (Partial LD: 5.04s, Full LD: $5.21 \mathrm{~s}, F(1,88)=1.78, p=.19)$.

$<$ Insert Figure 3 about here>> 
Steering reaction time (SRT). No statistically significant main effect of an error from the assistance (FW or MW) on the following lane departure and compared to no assistance SRTs was observed $F(7,88)=$ 1.38, $P=.22$ ). However, the lane departure onset significantly changed the SRTs (Partial LD: 0.92s, Full LD: $\left.1.14 \mathrm{~s}, F(1,88)=10.53, P<.01, \eta^{2}=0.11\right)$.

\subsubsection{Lateral position on the lane outside lane departure episodes}

None of assistance device unreliability, type of assistance error or onset factors significantly affected drivers' lateral position on the driving lane (respectively: $F(1,44)=0.91, p=.43 ; F(1,44)=1.77, p=.19$; $F(1,44)=0.21, p=.65)$. The cumulative surface around the lane centre outside lane departure episodes was significantly reduced in the presence of the assistance device compared to the no assistance condition (no assistance: $2406 \mathrm{~m}^{2}$, assistance: $2079 \mathrm{~m}^{2}, F(1,44)=44.9, p<.001, \eta^{2}=0.51$ ). See Table 1 for a complete overview of those results.

\subsection{Subjective assessment}

\subsubsection{Mental workload}

No statistically significant differences were revealed after the analysis of each of the six dimensions of the NASA-TLX (mental demand, physical demand, temporal demand, performance, effort and frustration). Subjective mental workload that included those six dimensions did not reveal any statistical effect either.

\subsubsection{Assistance acceptance}

No statistically significant differences were observed for the reliable, accurate, trustworthy, acceptable, pleasing dimensions regardless of factor considered.

The assistance device was judged more predictable, annoying and less agreeable with FW only compared to a combination of FW and MW (see Fig. 4; predictable: $F(1,44)=7.12, p<.02, \eta^{2}=0.14$; agreeable: $F(1,44)=4.4, p<.05, \eta^{2}=0.04$; annoying: $\left.F(1,44)=5.39, p<.03, \eta^{2}=0.11\right)$.

$<<$ Insert Figure 4 about here >>

The assistance device was also considered as more pleasing, agreeable and less annoying with lower assistance unreliability $(17 \%)$ than with higher unreliability $(33 \%)$ (see Fig. 5; pleasing: $F(1,44)=$ 4.3, $p<.05, \eta^{2}=0.09$; agreeable: $F(1,44)=7.67, p<.01, \eta^{2}=0.15$; annoying: $F(1,44)=6.78, p<.02, \eta^{2}=$ $0.13)$. 
$<$ Insert Figure 5 about here $>$

Finally, a Full LD warning onset was assessed as more agreeable and less annoying than a Partial LD warning onset (see Fig. 6; agreeable: $F(1,44)=4.21, p<.05, \eta^{2}=0.09$; annoying: $F(1,44)=$ $\left.5.68, p<.03, \eta^{2}=0.11\right)$.

$<<$ Insert Figure 6 about here >>

\section{Discussion}

This experiment investigated the combined influence of lane departure warning reliability and onset on driving behaviour and assistance acceptance. From a general perspective it was found that warnings improved drivers' responses to lane departure events. Least reliable assistance was found less effective in terms of time spent outside a lateral safety envelope than more reliable assistance (but still imperfect) especially with Full LD warnings. This result showed that warning reliability and onset interact at least for lane departure warning assistance device. When focusing on the specific lane departures immediately following a false or missed warning, no performance decrease compared to no assistance condition was observed in terms of time spent outside the driving lane. The assistance also helped drivers to improve general lane-keeping performances. In the presence of the assistance regardless of reliability level, nature of errors (false warning only or combined with missed warnings) and the warning onset drivers kept their vehicle closer to the lane centre than they did in the no assistance condition. Subjectively, drivers reported their preference for the most reliable assistance and for Full LD warnings. Subjective data match objective data as long as assistance reliability is considered. However, drivers tended to favour less effective Full LD warnings over more effective Partial LD warnings. Moreover, if participants observed the difference between false warnings alone or when combined with missed warnings, driving performances were not significantly affected by type of error.

The results obtained in the current experiment reinforce previous data indicating that LDW is a

yaluable lateral control assistance device that successfully improves driving performances during lane departure episodes (Hoc et al., 2006; Navarro et al., 2010, 2007; Suzuki \& Jansson, 2003). All previously collected results used perfectly reliable LDWS; therefore, the current experiment extends this result to imperfect LDWS with as many inaccuracies (FW or FW and $\mathrm{MW}$ ) as $17 \%$ and even $33 \%$. A trend regarding duration of lateral excursion has been observed between the two assistance unreliability levels. The less reliable assistance elicited longer mean duration of lateral excursion, indicating that the 
unreliability limit below which performance degradation occurred was about to be attained. This trend would confirm a prediction based on an analysis of 22 studies that indicated a reliability level of $70 \%$ as the point below which unreliable assistance would be worse than no assistance at all (Wickens \& Dixon, 2007).

The principle underlying LDW assistance efficiency may be broken down into two stages: (1) a faster reaction observed through steering wheel reaction time and previously reported with longitudinal control assistances (Bueno et al., 2012; Lee et al., 2002) and (2) a better situation diagnosis observed through attention redirection which improves situation diagnosis (Ho \& Spence, 2009; Ho, Tan, \& Spence, 2006) leading to shorter duration of lateral excursion.

The first stage of the LDW working principle is not sensitive to previous exposure. This was observed by the absence of a difference in steering reaction time for a lane departure regardless of previously non-warned, unnecessarily warned or correctly warned lane departures. Indeed, the warning was found to reduce steering reaction times in all of these situations in a similar way. This first stage of LDW is closely related to the warning itself and is not under the influence of previous events either at short or middle term. At this stage, lane departure warnings pre-activate drivers' motor response whatever the response may be. According to the idea that action expectancy improves performance (Posner, Snyder, \& Davidson, 1980), the warning urges drivers to do something. Warning onset, however, was found to modify steering reaction time from both a general point of view and immediately after a false or missed warning. Therefore, participants' responses on the steering wheel were not completely independent of the driving context. Partial LD onset induced faster steering reaction time than Full LD onset, as if drivers' action pre-activation might be adjusted depending on warning quality. More useful Partial LD warnings give rise to an optimum pre-activation. If steering reaction times were not sensitive to unreliable assistance, situation diagnosis (assessed by duration of lateral excursion) tended to be influenced by the reliability level, in particular with Full LD warnings. With inaccurate and too late warnings, the warning impact on situation diagnosis diminished and drivers probably favoured direct yisual analysis of the driving scene. Therefore when the warning was less predictable of the action to perform because of too many inaccuracies and too late delivery the quality of recovery manoeuvre was degraded. Those data are in accordance with previous results reporting performance decrease with unreliable assistance device (Abe et al., 2002; Bliss \& Acton, 2003; Enriquez \& MacLean, 2004; Maltz \& Shinar, 2004). Based on longitudinal control data, reliability effects on driving behaviours are not clear 
suggesting that imperfect warning effects are probably dependent on the driving context. As the second stage of the LDW working principle (i.e. situation diagnosis) is by nature also dependent on the context, results on reliability obtained here should be considered with caution because different results might be obtained in different contexts (such as road departures instead of lane departure, presence of opposite traffic during lane departure episodes or drowsiness induced lane departures for instance).

Drivers' reactions to False Warnings are not equivalent between a warning delivered by a Forward Collision Warning System (FCWS) and a LDWS. With FCWS FWs, drivers tend to release the accelerator pedal and therefore reduce speed as soon as a warning is delivered whatever the driving context. Then the situation diagnosis is performed and the vehicle speed adjusted accordingly. With LDW FWs, drivers did not undertake any action on the steering wheel or pedals as if they took the time to perform a complete situation diagnosis before modifying the vehicle control. This difference can be explained by the risk associated to the two different driving conditions under investigation. Indeed as far as longitudinal control is concerned, a speed reduction whatever the accuracy of the warning is not critical for safety. For lateral control however turning the steering wheel in reaction to a FW could lead to a real lane departure. Besides if drivers can categorize a warning as true or false based on a dynamic reference for both FCWS (estimation of the time-to-collision with the lead vehicle) and LDWS (estimation of the time-to-lane-crossing with the lane making), the devices are not equivalent because the associated driving tasks are not either. Drivers are solely responsible for the lateral position of their vehicle whereas the gap variations with a lead car are also under influence of the lead driver.

It is interesting and new to note, however, that our experiment revealed a negative cumulative effect of unreliable and lately warned lane departures (Full LD). Participants subjectively felt and declared a reliability difference between assistance unreliability of $17 \%$ and $33 \%$. The most reliable assistance device (17\% of incorrect warnings) was declared to be more pleasing, agreeable and less annoying than the less reliable device (33\% of incorrect warnings). But participants reported no interaction between warning onset and reliability.

Contrary to objective results, Full LD warnings were said to be more agreeable and less annoying than Partial LD warnings, in accordance with lower acceptance associated with early lane departure warnings already reported (Tijerina et al., 1996). The idea that the warning should be selected based on the situation's emergency (Werneke et al., 2013) can be extended here to the warning onset. It is assumed that the Partial LD warning as defined in this experiment, is an optimum between the risk it may 
be considered as a false warning and its benefits in time to adjust driving behaviour. The current results did not indicate that Partial LD warning signals, even if judged as annoying, were ignored by drivers (Abe \& Richardson, 2004, 2006). No performance degradation was recorded just after one or two Partial LD-triggered false warnings either. At the reverse and as shown with rear-end and forward collision warnings driving performances were improved even more with a Partial LD warning than with a Full LD warning (Abe \& Richardson, 2004, 2005, 2006; Lee et al., 2002).

Highly urgent sounds but also increased mental workload through annoyance increase were reported to elicit faster drivers responses (Wiese \& Lee, 2004). In our experiment the rumble strip sound used as a warning led to faster steering wheel responses in lane departure situations compared to a no warning situation, but no increase in mental workload. Drivers' subjective rating indicated that on average this warning was judged as moderately annoying (about 5 on a 0 to 10 scale). This confirms previous observations indicating that drivers are lukewarm about auditory LDW (Tijerina et al., 1996). In addition, participants observed a difference between unreliable assistance with false warning only or with a combination of false and missed warnings, attributing higher predictable and annoyance scores and lower agreeable scores to false warnings alone. Those results complied with lower acceptance and higher annoyance of false warnings (Maltz \& Shinar, 2004; Tijerina et al., 1996), but did not significantly affect driving performances.

In terms of behavioural adaptation outside lane departure situations, the assistance presence reduced participants' variability around the lane centre regardless of unreliability level, error type and warning onset. This result confirmed earlier similar observations (Rudin-Brown \& Noy, 2002).

If results obtained in this experiment improved our knowledge on assistance reliability and onset effects, further studies are required to extend our findings in a more complex everyday life context. For instance, warning efficiency has been revealed to be under the influence of the driver's cognitive state and, especially, its externally or internally driven distraction (e.g. Bueno et al., 2012). Future experiments are required to examine assistance reliability and warning onset effects using different means such as internal thoughts to distract drivers. Moreover, longer-term experiments are required to evaluate LWD inaccuracies over time. Especially because LDWS might be considered by drivers as a "not to awake device" due to the perception of the auditory warning as a criticism on driving behaviours that could interfere with a discussion or the music played in the vehicle for instance. Finally a matter of particular 
interest is to investigate how multiple assistance warning devices (LDW and FCW for instance), its inaccuracies and onsets interact when used together.

\section{Conclusion}

Partially reliable (17\% and 33\% of incorrect warnings) LDW assistance improved driving performances for subsequent lane departure situations overall. Even the lane departure situation immediately following a false or a missed warning can sometimes be improved compared to no assistance driving performances. In the worst-case scenario lane departure following assistance error result in similar performances to those observed without assistance. These data indicate that even less reliable LDW assistance can draw benefits. To optimise those benefits it appears that warning onset should be synchronised with imminent lane departure (Partial LD) and high low assistance unreliability (here 17\%). The assistance, even imperfectly reliable, also improves lane keeping with drivers staying closer to the lane centre outside lane departure episodes. From participants' subjective point of view, no mental workload and only some acceptance dimensions were modified depending on assistances characteristics. However, the best objective warning onset was judged as less agreeable and more annoying. In addition, drivers were sensitive to assistance unreliability level with the most reliable assistance judged as more pleasing, agreeable and less annoying. Subjective data tend to indicate that earlier onset and highly unreliable warnings might result in a decline in driving performance and/or lead drivers to switch off the assistance. To conclude it is important to stress that the results should not be interpreted as LDWS effectiveness whatever the reliability level of the device. Only two levels of LDWS unreliability were tested here and more importantly longitudinal studies are needed to evaluate slow changes in driving behaviours using imperfectly reliable LDWS. 
Acknowledgments

This study was funded by the French association "Fondation Sécurité Routière" (ACT project) and by the

LABEX CORTEX (ANR-11-LABX-0042) of Université de Lyon, within the program "Investissements

d'Avenir'” (ANR-11-IDEX-0007) operated by the French National Research Agency (ANR). The authors

are very grateful to Daniel Ndiaye, Bertrand Richard and Fabien Moreau for their technical support.

\section{References}

Abe, G., Itoh, M., \& Tanaka, K. (2002). Dynamics of drivers' trust in warning systems. In 15th IFAC World Congress (Vol. 5, p. 2002). Barcelona, Spain, July.

Abe, G., \& Richardson, J. (2004). The effect of alarm timing on driver behaviour: an investigation of differences in driver trust and response to alarms according to alarm timing. Transportation Research Part F: Traffic Psychology and Behaviour, 7(4), 307-322.

Abe, G., \& Richardson, J. (2005). The influence of alarm timing on braking response and driver trust in low speed driving. Safety Science, 43(9), 639-654.

Abe, G., \& Richardson, J. (2006). Alarm timing, trust and driver expectation for forward collision warning systems. Applied Ergonomics, 37(5), 577-586.

Ben-yaacov, A., Maltz, M., \& Shinar, D. (2002). Effects of an in-vehicle collision avoidance warning system on short- and long-term driving performance. Human Factors, 44(2), 335-342. doi:10.1518/0018720024497925

Bliss, J. P., \& Acton, S. A. (2003). Alarm mistrust in automobiles: how collision alarm reliability affects driving. Applied Ergonomics, 34(6), 499-509.

Bueno, M., Fabrigoule, C., Deleurence, P., Ndiaye, D., \& Fort, A. (2012). An electrophysiological study of the impact of a Forward Collision Warning System in a simulator driving task. Brain Research, 1470, 69-79. doi:10.1016/j.brainres.2012.06.027

Carson, J., \& Mannering, F. (2001). The effect of ice warning signs on ice-accident frequencies and severities. Accident Analysis \& Prevention, 33(1), 99-109. doi:10.1016/S0001-4575(00)00020-8

Charlton, S. G. (2006). Conspicuity, memorability, comprehension, and priming in road hazard warning signs. Accident Analysis \& Prevention, 38(3), 496-506. doi:10.1016/j.aap.2005.11.007

Charlton, S. G. (2007). The role of attention in horizontal curves: A comparison of advance warning, delineation, and road marking treatments. Accident Analysis \& Prevention, 39(5), 873-885. doi:10.1016/j.aap.2006.12.007

Dewar, R. (1988). Criteria for the design and evaluation of traffic sign symbols. Transportation Research Record: Journal of the Transportation Research Board, (1160), 1-6.

Dewar, R. (1993). Warning: Hazardous Road Signs Ahead. Ergonomics in Design: The Quarterly of Human Factors Applications, 1(3), 26-31. doi:10.1177/106480469300100310

Dingus, T. A., McGehee, D. V, Manakkal, N., Jahns, S. K., Carney, C., \& Hankey, J. M. (1997). Human factors field evaluation of automotive headway maintenance/collision warning devices. Human Factors: The Journal of the Human Factors and Ergonomics Society, 39(2), 216-229.

Edworthy, J., Stanton, N., \& Hellier, E. (1995). Warnings in research and practice. Ergonomics, 38, 2145-2154. doi:10.1080/00140139508925258

Enriquez, M., \& MacLean, K. E. (2004). Impact of haptic warning signal reliability in a time-and-safetycritical task. In Haptic Interfaces for Virtual Environment and Teleoperator Systems, 2004. HAPTICS'04. Proceedings. 12th International Symposium on (pp. 407-414). USA: IEEE.

Fisher, J. (1992). Testing the Effect of Road Traffic Signs' Informational Value on Driver Behavior. Human Factors: The Journal of the Human Factors and Ergonomics Society, 34(2), 231-237. 
Hart, S. G., \& Staveland, L. E. (1988). Human Mental Workload. Advances in Psychology, 52, 139-183. doi:10.1016/S0166-4115(08)62386-9

Ho, C., \& Spence, C. (2009). Using peripersonal warning signals to orient a driver's gaze. Human Factors, 51(4), 539-556. doi:10.1177/0018720809341735.

Ho, C., Tan, H. Z., \& Spence, C. (2006). The differential effect of vibrotactile and auditory cues on visual spatial attention. Ergonomics, 49(7), 724-738.

Hoc, J.-M., Mars, F., Milleville-Pennel, I., Jolly, É., Netto, M., \& Blosseville, J.-M. (2006). Humanmachine cooperation in car driving for lateral safety: delegation and mutual control. Le Travail Humain, 69(2), 153-182.

Hoc, J.-M., Young, M. S., \& Blosseville, J.-M. (2009). Cooperation between drivers and automation: implications for safety. Theoretical Issues in Ergonomics Science, 10(2), 135-160.

Itoh, M., \& Inagaki, T. (2014). Design and evaluation of steering protection for avoiding collisions during a lane change. Ergonomics, 57(3), 361-373.

Johansson, G., \& Backlund, F. (1970). Drivers and road signs. Ergonomics, 13(6), 749-759.

Kozak, K., Pohl, J., Birk, W., Greenberg, J., Artz, B., Blommer, M., ... Curry, R. (2006). Evaluation of lane departure warnings for drowsy drivers. In Proceedings of the Human Factors and Ergonomics Society Annual Meeting (Vol. 50, pp. 2400-2404). Sage Publications.

Lee, J. D., McGehee, D., Brown, T. L., \& Reyes, M. L. (2002). Collision warning timing, driver distraction, and driver response to imminent rear-end collisions in a high-fidelity driving simulator. Human Factors, 44(2), 314-334.

Lees, M. N., \& Lee, J. D. (2007). The influence of distraction and driving context on driver response to imperfect collision warning systems. Ergonomics, 50(8), 1264-1286.

Lehto, M. R. (2000). Designing warning signs and warning labels: Part II — Scientific basis for initial guidelines. In Elsevier Ergonomics Book Series (Vol. 1, pp. 257-280). doi:10.1016/S1572347X(00)80021-X

Lété, B., Sprenger-Charolles, L., \& Colé, P. (2004). MANULEX: A grade-level lexical database from French elementary school readers, Behavior Research Methods, 36(1), 156-166.

Macdonald, W. A., \& Hoffmann, E. R. (1991). Drivers' awareness of traffic sign information. Ergonomics, 34(5), 585-612.

Maltz, M., \& Shinar, D. (2004). Imperfect in-vehicle collision avoidance warning systems can aid distracted drivers. Human Factors, 46(2), 357-366.

Maltz, M., \& Shinar, D. (2007). Imperfect in-vehicle collision avoidance warning systems can aid distracted drivers. Transportation Research Part F: Traffic Psychology and Behaviour, 10(4), 345357.

McGehee, D. V, Brown, T. L., Lee, J. D., \& Wilson, T. B. (2002). Effect of warning timing on collision avoidance behavior in a stationary lead vehicle scenario. Transportation Research Record: Journal of the Transportation Research Board, 1803(1), 1-6.

Navarro, J., Mars, F., Forzy, J.-F., El-Jaafari, M., \& Hoc, J.-M. (2010). Objective and subjective evaluation of motor priming and warning systems applied to lateral control assistance. Accident; Analysis and Prevention, 42(3), 904-12. doi:10.1016/j.aap.2009.07.008

Navarro, J., Mars, F., \& Hoc, J.-M. (2007). Lateral control assistance for car drivers: a comparison of motor priming and warning systems. Human Factors, 49(5), 950-60.

Navarro, J., Mars, F., \& Young, M. S. (2011). Lateral control assistance in car driving: classification, review and future prospects. IET Intelligent Transport Systems, 5(3), 207. doi:10.1049/ietits. 2010.0087

Parasuraman, R., Hancock, P. A., \& Olofinboba, O. (1997). Alarm effectiveness in driver-centred collision-warning systems. Ergonomics, 40(3), 390-399.

Posner, M. I., Snyder, C. R., \& Davidson, B. J. (1980). Attention and the detection of signals. Journal of 
Experimental Psychology: General, 109(2), 160.

Reagan, I. J., \& Bliss, J. P. (2013). Perceived mental workload, trust, and acceptance resulting from exposure to advisory and incentive based intelligent speed adaptation systems. Transportation Research Part F: Traffic Psychology and Behaviour, 21, 14-29. doi:10.1016/j.trf.2013.07.005

Rudin-Brown, C. M., \& Noy, I. Y. (2002). Investigation of behavioral adaptation to lane departure warnings. Transportation Research Record: Journal of the Transportation Research Board, 1803(1), 30-37.

Shinar, D. (1978). Psychology on the Road. The Human Factor in Traffic Safety (Wiley.). New York.

Shladover, S. E. (1995). Review of the state of development of advanced vehicle control systems (AVCS). Vehicle System Dynamics, 24(6-7), 551-595.

Stanton, N. A., Young, M. S., Walker, G. H., Turner, H., \& Randle, S. (2001). Automating the driver's control tasks. International Journal of Cognitive Ergonomics, 5(3), 221-236. doi:10.1207/S15327566IJCE0503_5

Suzuki, K., \& Jansson, H. (2003). An analysis of driver's steering behaviour during auditory or haptic warnings for the designing of lane departure warning system. JSAE Review, 24, 65-70. doi:10.1016/S0389-4304(02)00247-3

Tango, F., \& Montanari, R. (2006). Shaping the drivers' interaction: how the new vehicle systems match the technological requirements and the human needs. Cognition, Technology \& Work, 8(3), 215226.

Tijerina, L., Jackson, J. L., Pomerleau, D. A., Romano, R. A., \& Petersen, A. D. (1996). Driving simulator tests of lane departure collision avoidance systems. In Intelligent Transportation: Realizing the Benefits. Proceedings of the 1996 Annual Meeting of ITS America.

Vahidi, A., \& Eskandarian, A. (2003). Research advances in intelligent collision avoidance and adaptive cruise control. Intelligent Transportation Systems, IEEE Transactions on, 4(3), 143-153.

Werneke, J., Kleen, A., \& Vollrath, M. (2013). Perfect Timing: Urgency, Not Driving Situations, Influence the Best Timing to Activate Warnings. Human Factors, 56, 249-259. doi: $10.1177 / 0018720813490728$

Wickens, C. D., \& Dixon, S. R. (2007). The benefits of imperfect diagnostic automation: A synthesis of the literature. Theoretical Issues in Ergonomics Science, 8(3), 201-212.

Wiese, E. E., \& Lee, J. D. (2004). Auditory alerts for in-vehicle information systems: the effects of temporal conflict and sound parameters on driver attitudes and performance. Ergonomics, 47, 965986. doi:10.1177/154193120104502315

World Health Organization. (2013). Global status report on road safety. Geneva: World Health Organization.

doi:http://www,who.int/violence_injury_prevention/road_safety_status/2013/en/index.html

Young, M. S., Stanton, N. A., \& Harris, D. (2007). Driving automation: learning from aviation about design philosophies. International Journal of Vehicle Design, 45(3), 323-338.

Ziegler, W., Franke, U., Renner, G., \& Kühnle, A. (1995). Computer vision on the road: A lane departure and drowsy driver warning system. SAE Technical Paper. 
Figure 1:
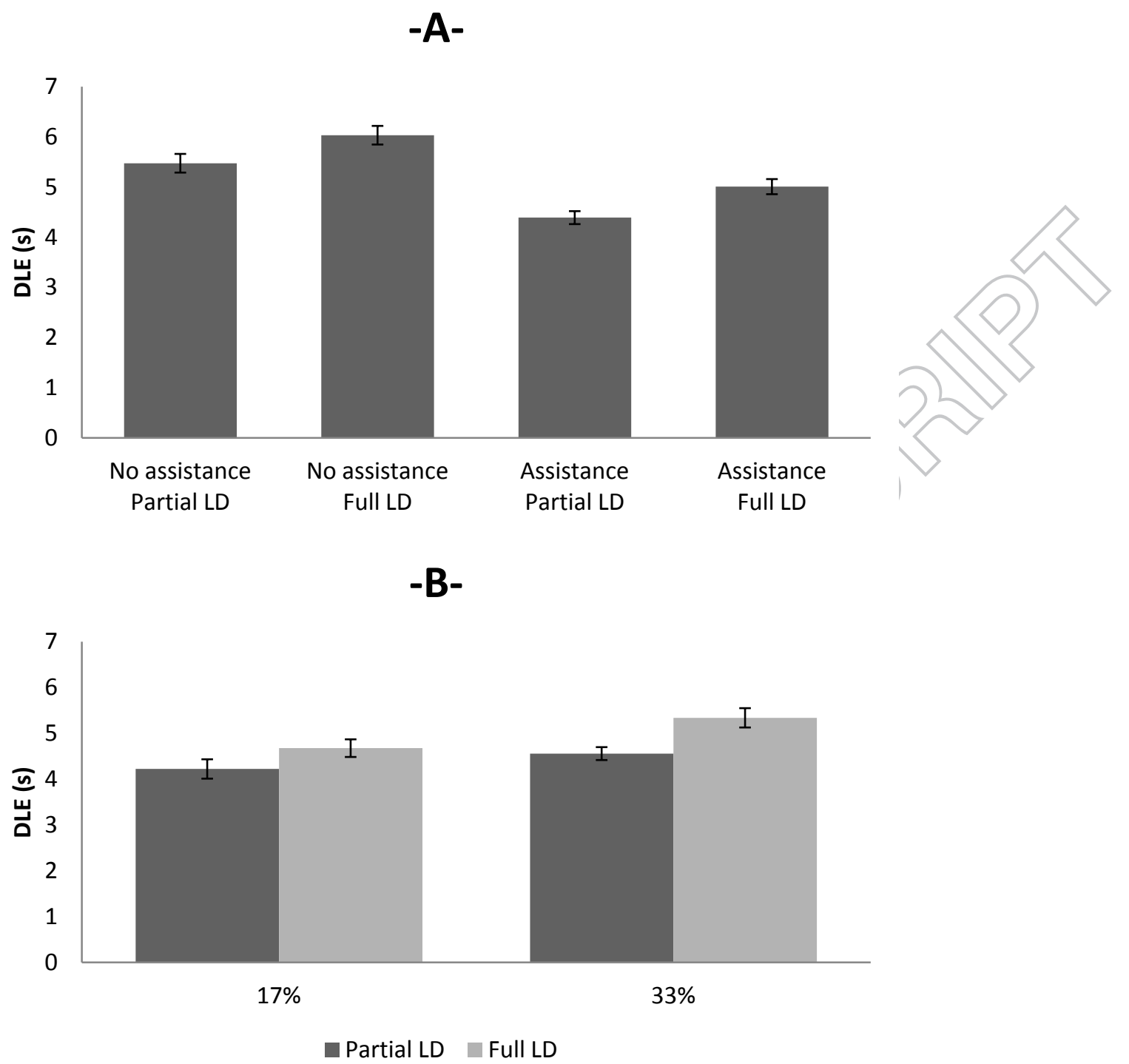
Figure 2:

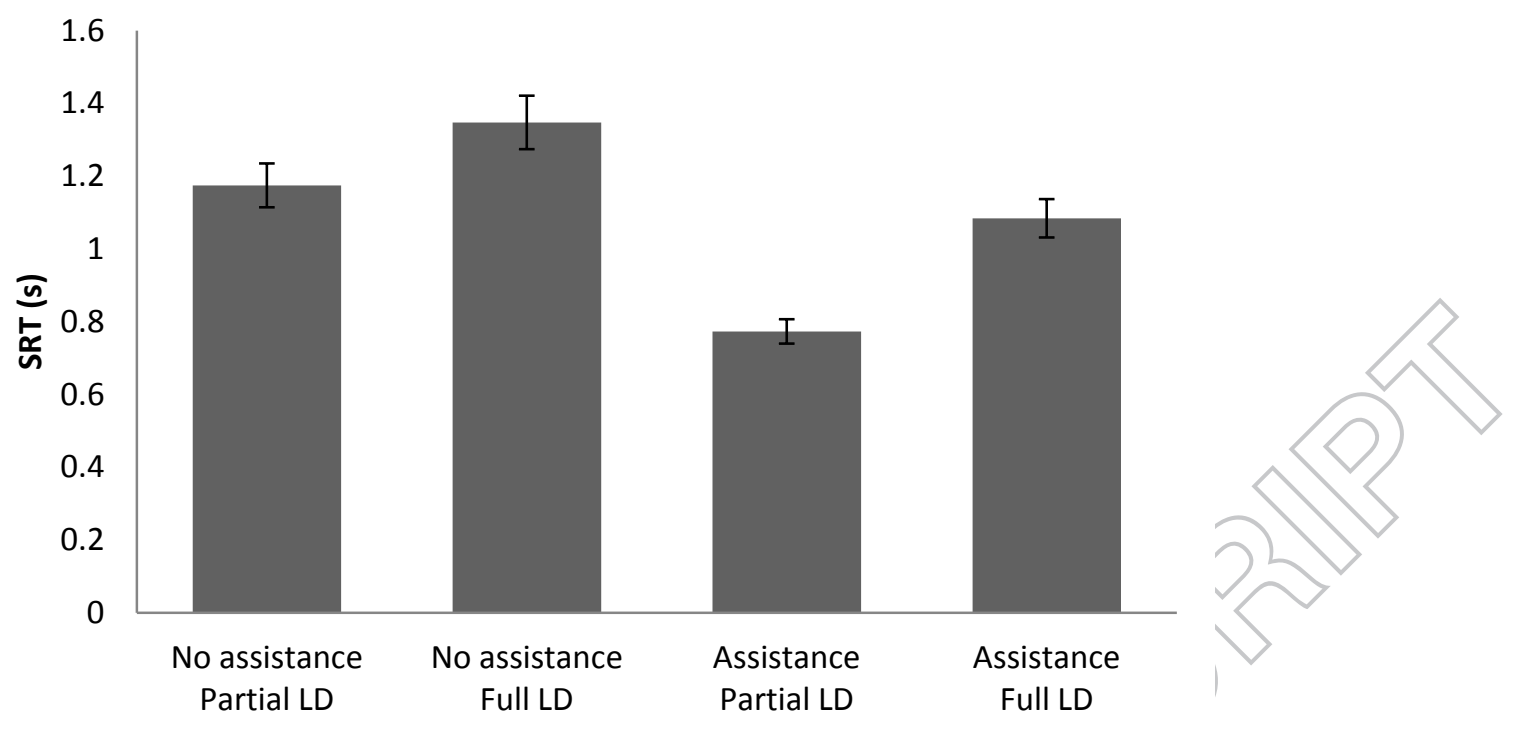


Figure 3:

No assistance

MW 1 in 33\% FW and MW group

FW 1 in 33\% FW and MW group

FW 2 in 33\% FW group

FW 1 in 33\% FW group

FW 1 in 17\% FW and MW group

MW 1 in 17\% FW and MW group

FW 1 in 17\% FW group

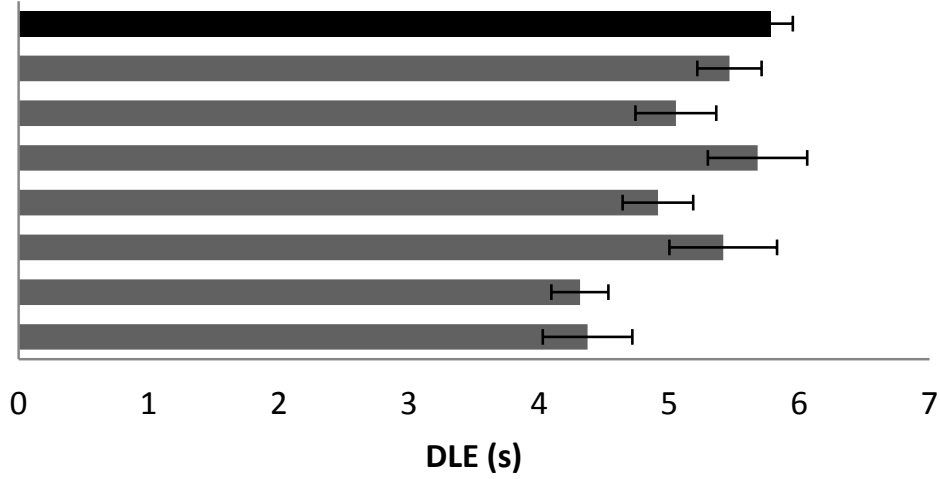

DLE (s) 
Figure 4:

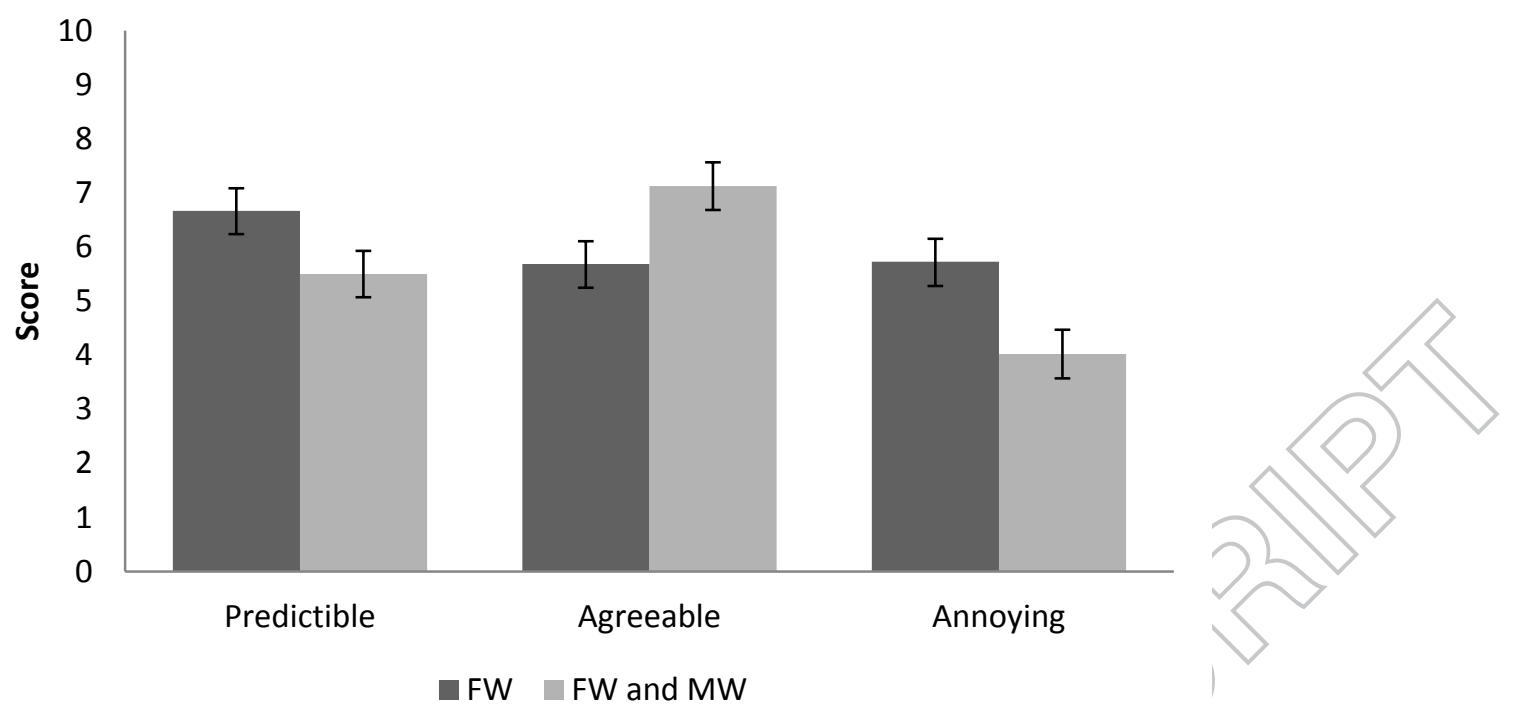


Figure 5:

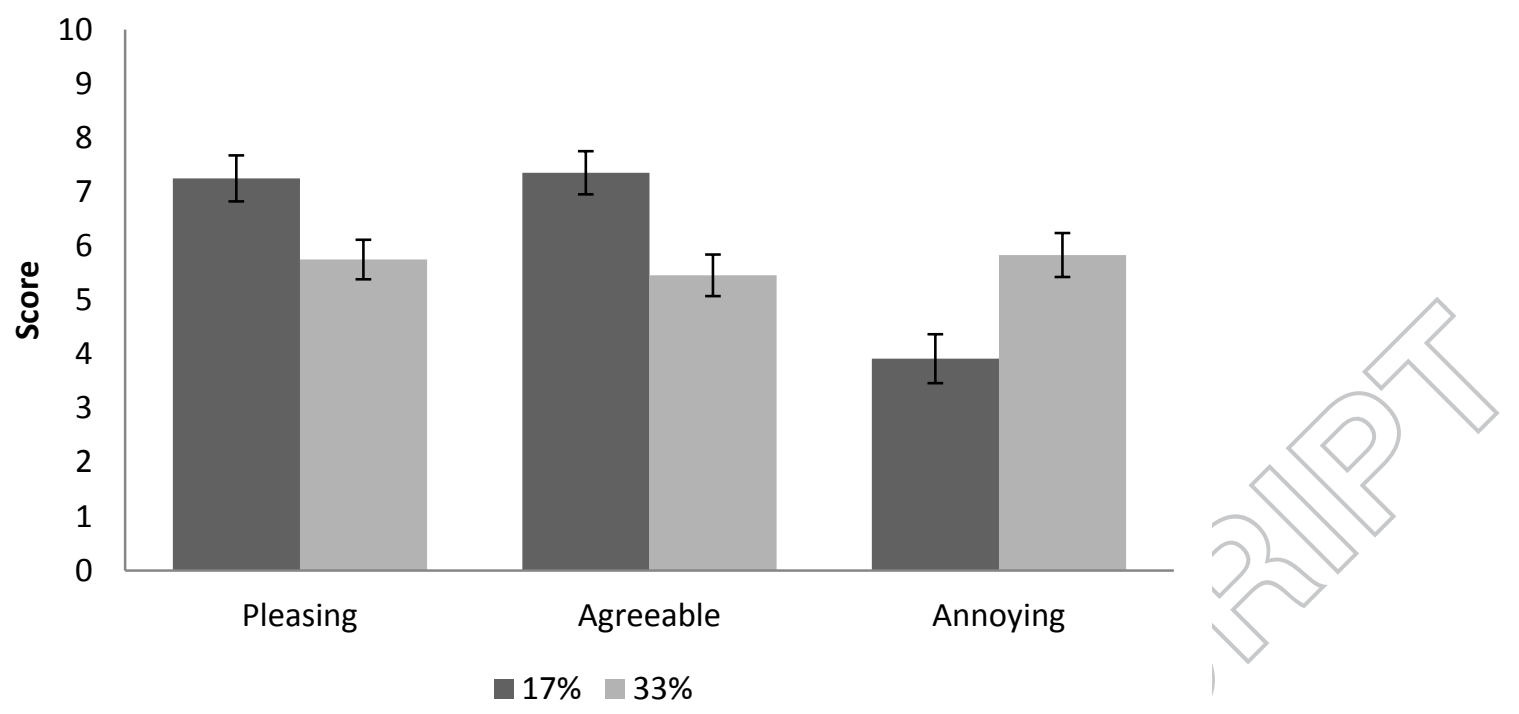


Figure 6:

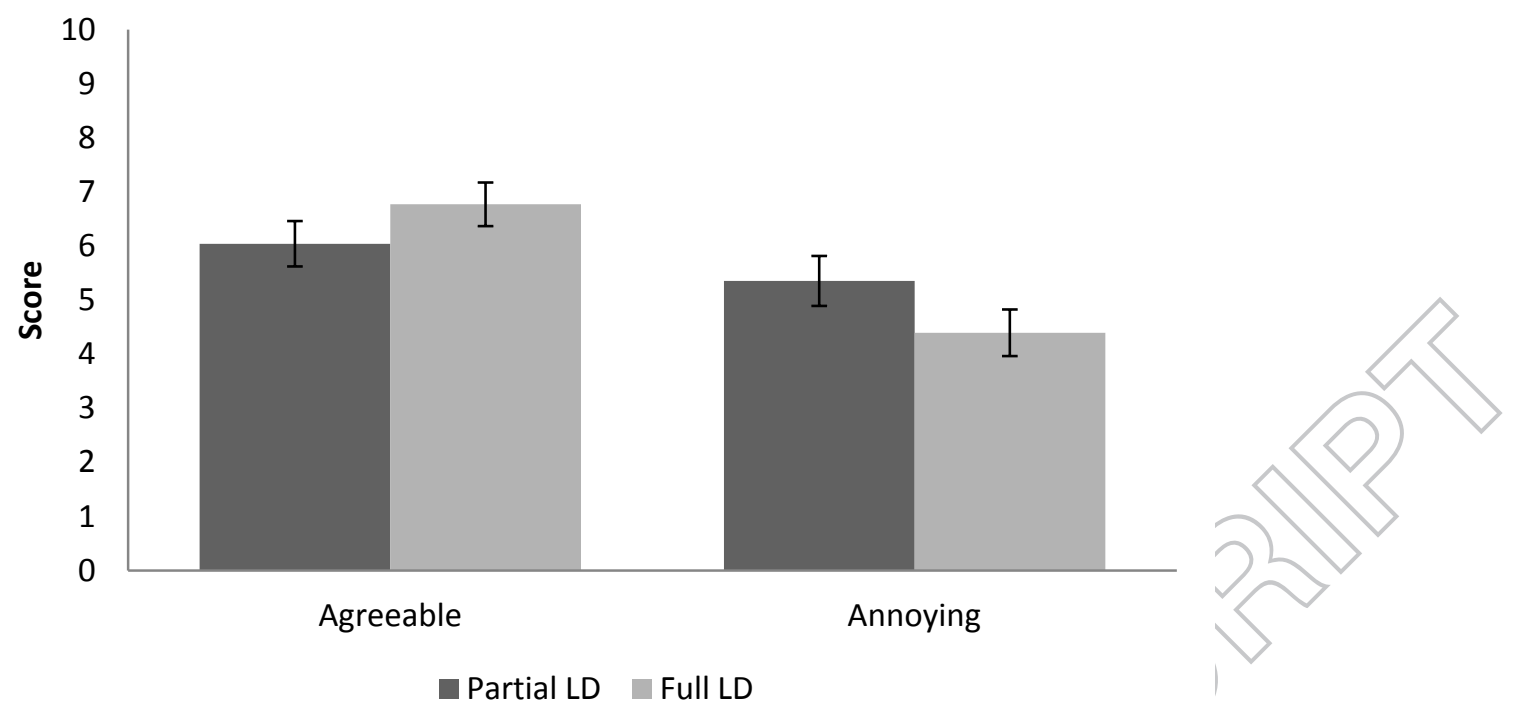


Table 1:

\begin{tabular}{|l|l|l|l|l|}
\cline { 2 - 5 } \multicolumn{1}{c|}{} & $\begin{array}{l}\text { Scenario 1 } \\
\text { (NA, Partial LD) }\end{array}$ & $\begin{array}{l}\text { Scenario 2 } \\
\text { (NA, Full LD) }\end{array}$ & $\begin{array}{l}\text { Scenarios 3 } \\
\text { (A, Partial LD) }\end{array}$ & $\begin{array}{l}\text { Scenario 4 } \\
\text { (A, Full LD) }\end{array}$ \\
\hline Group 1 & DLE: $4.97 \pm 0.46$ & DLE: $5.11 \pm 0.35$ & DLE: $4.09 \pm 0.35$ & DLE: $4.50 \pm 0.32$ \\
(33\% unreliable, & SRT: $0.99 \pm 0.14$ & SRT: $1.15 \pm 0.14$ & SRT: $0.81 \pm 0.09$ & SRT: $1.02 \pm 0.12$ \\
FW) & Lpos: $2269 \pm 150$ & Lpos: $2165 \pm 160$ & Lpos: $1897 \pm 159$ & Lpos: $1733 \pm 115$ \\
\hline Group 2 & DLE: $5.59 \pm 0.31$ & DLE: $6.15 \pm 0.25$ & DLE: $4.35 \pm 0.23$ & DLE: $4.85 \pm 0.20$ \\
(33\% unreliable, & SRT: $1.24 \pm 0.09$ & SRT: $1.44 \pm 0.15$ & SRT: $0.68 \pm 0.05$ & SRT: $1.10 \pm 0.09$ \\
FW + MW) & Lpos: $2398 \pm 263$ & Lpos: $2463 \pm 188$ & Lpos: $2119 \pm 175$ & Lpos: $2109 \pm 191$ \\
\hline Group 3 & DLE: $5.51 \pm 0.30$ & DLE: $6.12 \pm 0.34$ & DLE: $4.50 \pm 0.19$ & DLE: $5.35 \pm 0.31$ \\
(17\% unreliable, & SRT: $1.28 \pm 0.13$ & SRT: $1.35 \pm 0.15$ & SRT: $0.89 \pm 0.05$ & SRT: $1.14 \pm 0.11$ \\
FW) & Lpos: $2367 \pm 212$ & Lpos: $2422 \pm 231$ & Lpos: $1854 \pm 165$ & Lpos: $2139 \pm 199$ \\
\hline Group 4 & DLE: $5.82 \pm 0.35$ & DLE: $6.76 \pm 0.36$ & DLE: $4.62 \pm 0.20$ & DLE: $5.33 \pm 0.29$ \\
(17\% unreliable, & SRT: $1.19 \pm 0.09$ & SRT: $1.45 \pm 0.14$ & SRT: $0.72 \pm 0.05$ & SRT: $1.08 \pm 0.09$ \\
FW + MW) & Lpos: $2561 \pm 296$ & Lpos: $2605 \pm 308$ & Lpos: $2393 \pm 212$ & Lpos: $2391 \pm 276$ \\
\hline
\end{tabular}


Figure and Table Caption:

Figure 1. Impact of assistance errors on subsequent lane departures. (A) Mean duration of lateral excursion depending on assistance (assistance, no assistance) and onset factors (Partial LD, Full LD). (B) Mean duration of lateral excursion depending on assistance unreliability $(17 \%, 33 \%)$ and onset factors (Partial LD, Full LD). Error bars represent standard errors.

Figure 2. Impact of assistance errors on subsequent lane departures. Mean steering reaction times depending on assistance (assistance, no assistance) and onset factors (Partial LD, Full LD). Error bars represent standard errors.

Figure 3. Mean duration of lateral excursion for the lane departure following a false (FW) or a missed (MW) warning. Error bars represent standard errors.

Figure 4. Mean subjective evaluation of the assistance device with False Warnings (FW) only and with False Warning and Missed Warnings (FW and MW) for the predictable, agreeable and annoying dimensions of acceptance. Error bars represent standard errors.

Figure 5. Mean subjective evaluation of the assistance device with $17 \%$ and $33 \%$ of unreliability for the pleasing, agreeable and annoying dimensions of acceptance. Error bars represent standard errors.

Figure 6. Mean subjective evaluation of the assistance device with Partial LD and Full LD onsets for the agreeable and annoying dimensions of acceptance. Error bars represent standard errors.

Table 1. Overview table of the results crossing driving scenarios with participants groups for the impact of False Warnings and False Warning plus Missed Warnings on subsequent lane departures on mean Duration of Lateral Excursion (DLE in seconds), mean Steering Reaction Time (SRT in seconds), and on the mean lateral position on the lane outside lane departure episodes (Lpos in $\mathrm{m}^{2}$ ). 